\title{
Pairing renormalization and regularization within the local density approximation
}

\author{
P.J. Borycki, ${ }^{1,2}$ J. Dobaczewski, ${ }^{1,3,4,5}$ W. Nazarewicz, ${ }^{1,3,4}$ and M.V. Stoitsov ${ }^{1,3,5,6}$ \\ ${ }^{1}$ Department of Physics $\&$ Astronomy, University of Tennessee, Knoxville, Tennessee 37996, USA \\ ${ }^{2}$ Institute of Physics, Warsaw University of Technology, ul. Koszykowa 75, 00-662 Warsaw, Poland \\ ${ }^{3}$ Physics Division, Oak Ridge National Laboratory, \\ P.O. Box 2008, Oak Ridge, Tennessee 37831, USA \\ ${ }^{4}$ Institute of Theoretical Physics, Warsaw University, ul. Hoża 69, 00-681 Warsaw, Poland \\ ${ }^{5}$ Joint Institute for Heavy-Ion Research, Oak Ridge, Tennessee 37831, USA \\ ${ }^{6}$ Institute of Nuclear Research and Nuclear Energy, \\ Bulgarian Academy of Sciences, Sofia-1784, Bulgaria
}

(Dated: February 18, 2018)

\begin{abstract}
We discuss methods used in mean-field theories to treat pairing correlations within the local density approximation. Pairing renormalization and regularization procedures are compared in spherical and deformed nuclei. Both prescriptions give fairly similar results, although the theoretical motivation, simplicity, and stability of the regularization procedure makes it a method of choice for future applications.
\end{abstract}

PACS numbers: 21.60.-n,21.60.Jz,31.15.Ew,21.10.Dr

\section{INTRODUCTION}

One of the main goals of the low energy nuclear theory is to build a comprehensive microscopic framework, in which nuclear bulk properties, excitations, and low energy reactions can be described. For medium-mass and heavy nuclei, self-consistent methods based on the Density Functional Theory (DFT) 1, 2] have already achieved a level of precision that allows for analysis of experimental data for a wide range of properties of nuclei throughout the chart of the nuclides. For example, the self-consistent Hartree-Fock-Bogoliubov (HFB) models based on the Skyrme energy functionals [3, 4, 5] are able nowadays to reproduce nuclear masses with an rms error of about $700 \mathrm{keV}$ [6, 7]. The development of a universal nuclear density functional, however, still requires a better understanding and improved description of the density dependence, isospin effects, pairing force, manybody correlations, and symmetry restoration.

Nuclear pairing is an important ingredient of the nuclear density functional, and it becomes crucial for open shell nuclei, in particular weakly bound systems, where the effects of coupling to continuum become significant [8, 9]. In this case, the BCS model is not adequate [8] and the fully self-consistent HFB approach must be used.

In most HFB applications, pairing interaction is assumed to be either in the form of the finite range Gogny force [10] or the zero-range, possibly density-dependent, delta force [8, 9, 11]. Gogny interaction in the pairing channel can be viewed as a regularized contact interaction, with regularization fixed through the finite range. The resulting pairing field is, however, nonlocal.

Calculations using the contact interaction are numerically simpler, but one has to apply a cutoff procedure within a given space of single-particle (s.p.) states [8, 12]. When the dimension of this space increases, the pairing gap diverges for any given strength of the interaction. Therefore, the pairing strength has to be readjusted for each s.p. space. Thus the energy cutoff and the pairing strength together define the pairing interaction, and this definition can be understood as a phenomenological introduction of finite range [8, 13]. Such a procedure is usually referred to as the renormalization of the contact pairing force. It is performed in the spirit of the effective field theory, whereupon contact interactions are used to describe low energy phenomena while the coupling constants are readjusted for any given energy cutoff to take into account neglected high energy effects.

The renormalization procedure for the zero-range pairing interactions has been explored in Ref. [8] using the numerical solutions of the HFB equations. It has been shown that by renormalizing the pairing strength for each value of the cutoff energy one practically eliminates the dependence of the HFB energy on the cutoff parameter.

Recently, the issue of contact pairing force has been addressed in Refs. 14, 15, 16, 17, 18, 19, 20, 21, 22], suggesting that the renormalization procedure can be replaced by a regularization scheme which removes the cutoff energy dependence of the pairing strength. In subsequent papers, this regularization scheme has been applied to properties of the infinite nuclear matter 19], spherical nuclei [21, 23], and trapped fermionic atoms [16, 24].

In this study, we investigate the stability of the regularization scheme with respect to the cutoff energy for both spherical and deformed nuclei. Differences between the HFB results emerging from the pairing renormalization and pairing regularization procedures are analyzed.

The HFB and Skyrme HFB formalisms have been explained in great detail in many papers (see, e.g., Refs. 12, 25]). The notation used in the present paper is consistent with that of Refs. [8, 12, 26]. This work is organized as follows. Sec. III gives a brief introduction to the pairing renormalization and regularization schemes. In Sect. III we explain the numerical framework used. The comparison between pairing regularization and renormalization techniques, studied for a large set of spherical and 
deformed nuclei, is discussed in Sec.IV Finally, the summary and conclusions are given in Sec. D

\section{THE CUTOFF PROCEDURES}

\section{A. Pairing Renormalization Procedure}

Within the HFB theory, the energy cutoff can be applied either to the s.p. or to the quasiparticle spectrum. The first option is used when the HFB equations are solved within a restricted s.p. space. However, the s.p. energies play only an auxiliary role in the HFB method, and the cutoff applied to the quasiparticle spectrum is more justified. This is done by using the so-called equivalent s.p. spectrum [12]:

$$
\bar{e}_{n}=\left(1-2 P_{n}\right) E_{n}+\mu,
$$

where $E_{n}$ is the quasiparticle energy and $P_{n}$ denotes the norm of the lower component of the HFB wave function.

Due to the similarity between $\bar{e}_{n}$ and the s.p. energies, one takes into account only those quasiparticle states for which $\bar{e}_{n}$ is less than the assumed cutoff energy $\epsilon_{\text {cut }}$.

It was shown [8] that for a fixed pairing strength the pairing energies depend significantly on the energy cutoff. Within the renormalization scheme employed in this work, we use the prescription of adjusting the pairing strength to obtain a fixed average neutron pairing gap [12],

$$
\bar{\Delta}=-\frac{1}{N} \int d^{3} \mathbf{r} d^{3} \mathbf{r}^{\prime} \sum_{\sigma \sigma^{\prime}} \tilde{h}\left(\mathbf{r} \sigma, \mathbf{r}^{\prime} \sigma^{\prime}\right) \rho\left(\mathbf{r}^{\prime} \sigma^{\prime}, \mathbf{r} \sigma\right),
$$

in ${ }^{120} \mathrm{Sn}$ equal to the experimental value of $1.245 \mathrm{MeV}$. In Eq. (2.2) $N$ is the number of particles, $\rho$ is the particle density, and $\tilde{h}$ is the pairing Hamiltonian (see Appendix A).

Such a procedure almost eliminates the dependence of the HFB energy on the cutoff [8].

\section{B. Pairing Regularization Procedure}

Using the HFB equations and properties of the Bogoliubov transformation (see appendix A for details), one concludes that the local abnormal density $\tilde{\rho}$ has a singular behavior when $\epsilon_{\text {cut }} \rightarrow \infty$. The standard regularization technique is to remove the divergent part and define the regularized local abnormal density $\tilde{\rho}_{r}(\mathbf{r})$ as

$$
\tilde{\rho}_{r}(\mathbf{r})=\lim _{\mathbf{x} \rightarrow 0}[\tilde{\rho}(\mathbf{r}-\mathbf{x} / 2, \mathbf{r}+\mathbf{x} / 2)-f(\mathbf{r}, \mathbf{x})],
$$

where $f$ is a regulator which removes the divergence at $\mathbf{x}=0$.

For cutoff energies high enough, one can explicitly identify 17, 18, 19] components generating divergence in the abnormal density (see, e.g., Eq. (21) of Ref. [18]):

$f(\mathbf{r}, \mathbf{x})=\frac{i \tilde{h}(\mathbf{r}) M^{*}(\mathbf{r}) k_{F}(\mathbf{r})}{4 \pi \hbar^{2}}+\frac{\tilde{h}(\mathbf{r})}{2} G_{\mu}(\mathbf{r}+\mathbf{x} / 2, \mathbf{r}-\mathbf{x} / 2)$,

where $G_{\mu}$ is the s.p. Green's function at the Fermi level $\mu$ in the truncated space, $M^{*}$ is the effective mass, and the Fermi momentum is

$$
k_{F}(\mathbf{r})=\frac{\sqrt{2 M^{*}(\mathbf{r})}}{\hbar} \sqrt{\mu-U(\mathbf{r})}
$$

with $U$ being the self-consistent mean-field potential.

The first term in Eq. (2.4) comes from the MacLaurin expansion with respect to $\mathbf{x}$; it guarantees that the regularization procedure does not introduce any constant term to the abnormal density and that $f(\mathbf{r}, \mathbf{x})$ solely represents the divergent part of $\tilde{\rho}$.

Using the Thomas-Fermi approximation, the local s.p. Green's function $G_{\mu}(\mathbf{r}):=G_{\mu}(\mathbf{r}, \mathbf{r})$ becomes 18,19$]$

$$
G_{\mu}(\mathbf{r})=\frac{1}{2 \pi^{2}} \lim _{\gamma \rightarrow 0} \int_{0}^{k_{\text {cut }}(\mathbf{r})} \frac{k^{2} d k}{\mu-\frac{\hbar^{2} k^{2}}{2 M^{*}(\mathbf{r})}-U(\mathbf{r})+i \gamma}
$$

where the cutoff momentum is given by:

$$
k_{\text {cut }}(\mathbf{r})=\frac{\sqrt{2 M^{*}(\mathbf{r})}}{\hbar} \sqrt{\epsilon_{\text {cut }}+\mu-U(\mathbf{r})} .
$$

The regularized pairing Hamiltonian and the pairing energy density may be written, respectively, as [18]:

$$
\begin{aligned}
\tilde{h}(\mathbf{r}) & =g(\mathbf{r}) \tilde{\rho}_{r}(\mathbf{r})=g_{\text {eff }}(\mathbf{r}) \tilde{\rho}(\mathbf{r}) \\
\mathcal{H}_{\text {pair }}(\mathbf{r}) & =\frac{1}{2} g_{\text {eff }}(\mathbf{r}) \tilde{\rho}(\mathbf{r})^{2}
\end{aligned}
$$

where the effective pairing strength [17, 18, 19],

$$
g_{e f f}(\mathbf{r})=\left(\frac{1}{g(\mathbf{r})}+\frac{G_{\mu}(\mathbf{r})}{2}+\frac{i M^{*}(\mathbf{r}) k_{F}(\mathbf{r})}{4 \pi \hbar^{2}}\right)^{-1}
$$

after calculating integral (2.6), can be expressed in the form: 


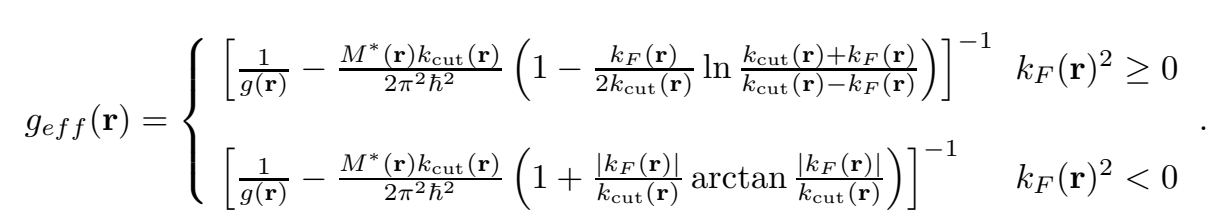

In this regularization scheme, only the Green's function is calculated using the Thomas-Fermi approximation. The densities, potentials, and chemical potential are determined self-consistently within the HFB theory. Consequently, the Fermi momentum (2.5) depends on microscopic HFB quantities. According to the sign of $k_{F}^{2}$, one of the expressions (2.11) is used.

In Ref. [20] a different regularization scheme has been proposed that involves truncation below and above the Fermi level. However, the HFB calculations in the quasiparticle basis should be performed for a high cutoff energy of $50 \mathrm{MeV}$ and higher [8]. Since the magnitude of the self-consistent mean field $U$ is also about $50 \mathrm{MeV}$, for such a high cutoff energy both methods are equivalent. The Thomas-Fermi approximation requires that, in order to obtain results independent of $\epsilon_{\text {cut }}$, its value should be high enough for $k_{\text {cut }}$ to be real everywhere.

Through the density dependence of $g_{\text {eff }}, k_{\text {cut }}$, and $k_{F}$, there appear rearrangement terms in the self-consistent mean-field potential:

$$
\begin{aligned}
& \frac{\delta \mathcal{H}_{\text {pair }}}{\delta \rho}=\frac{\delta g_{\text {eff }}}{\delta \rho} \tilde{\rho}^{2}=\tilde{\rho}^{2} \times \\
& \quad \times\left(\frac{\partial g_{\text {eff }}}{\partial g} \frac{\delta g}{\delta \rho}+\frac{\partial g_{\text {eff }}}{\partial k_{F}} \frac{\delta k_{F}}{\delta \rho}+\frac{\partial g_{\text {eff }}}{\partial k_{\text {cut }}} \frac{\delta k_{\text {cut }}}{\delta \rho}\right)
\end{aligned}
$$

The first term in Eq. (2.12) is similar to the usual rearrangement term, while the other two terms associated with the regularization procedure are entirely new. It is easy to check that all the terms appearing in Eq. (2.12) are continuous at the classical turning point $k_{F}(\mathbf{r})=0$.

In Eq. 2.9), the pairing energy density is divergent with respect to the cutoff energy. However, the pairing energy itself is not an observable, and in order for the energy density functional to be independent of the cutoff, other terms have to cancel out this divergence. As discussed in Refs. 14, 15, 17, 18, the kinetic energy density $\tau$ has the same type of divergence as the abnormal density $\tilde{\rho}$, and the sum

$$
\mathcal{H}_{\text {kin }+ \text { pair }}(\mathbf{r})=-\frac{\hbar^{2}}{2 M^{*}(\mathbf{r})} \tau+\frac{1}{2} g_{\text {eff }}(\mathbf{r}) \tilde{\rho}^{2}(\mathbf{r})
$$

does converge.

Various contributions to the total HFB energy as functions of the cutoff energy are shown in Fig. 1 The total energy is stable with respect to $\epsilon_{\text {cut }}$, although some of the components of the total energy vary significantly. As expected from Eq. (2.13), two terms exhibiting large fluctuations are the kinetic term (with vari-

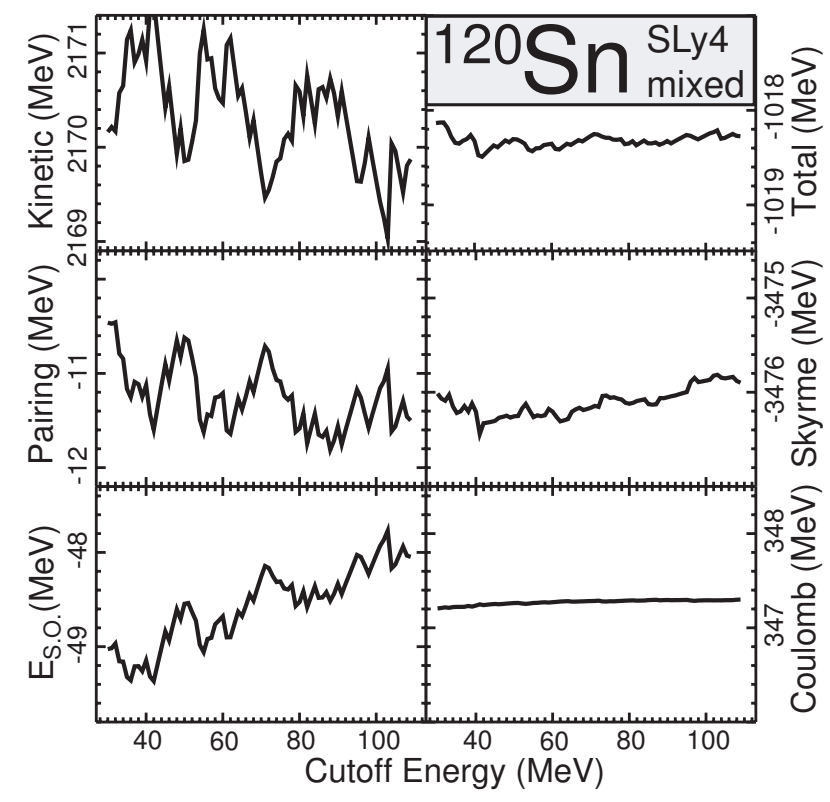

FIG. 1: Various contributions to the HFB energy for ${ }^{120} \mathrm{Sn}$ as a function of $\epsilon_{\text {cut }}$. Calculations are performed using the SLy4 Skyrme functional and mixed pairing interaction (3.1).

ations of about $2 \mathrm{MeV}$ ) and pairing term (with variations of about $1.3 \mathrm{MeV}$ ). Also, the momentum-dependent spin-orbit term, $E_{S . O}$., has significant variations of about $1 \mathrm{MeV}$. On the other hand, Skyrme and Coulomb energies are fairly stable with respect to $\epsilon_{\text {cut }}$.

\section{NUMERICAL IMPLEMENTATION}

\section{A. Numerical Framework}

As the pairing renormalization and regularization procedures remove the divergent part of the abnormal density in a different way, one can expect some numerical differences between both methods. In order to compare their results, we have performed numerical calculations using two numerical codes solving the HFB equations:

- HFBRAD 27] - solves the HFB equations in the spherically symmetric coordinate basis. The maximum angular momenta used in calculations were $j_{\max }=39 / 2$ for neutrons and $j_{\max }=25 / 2$ for protons. 


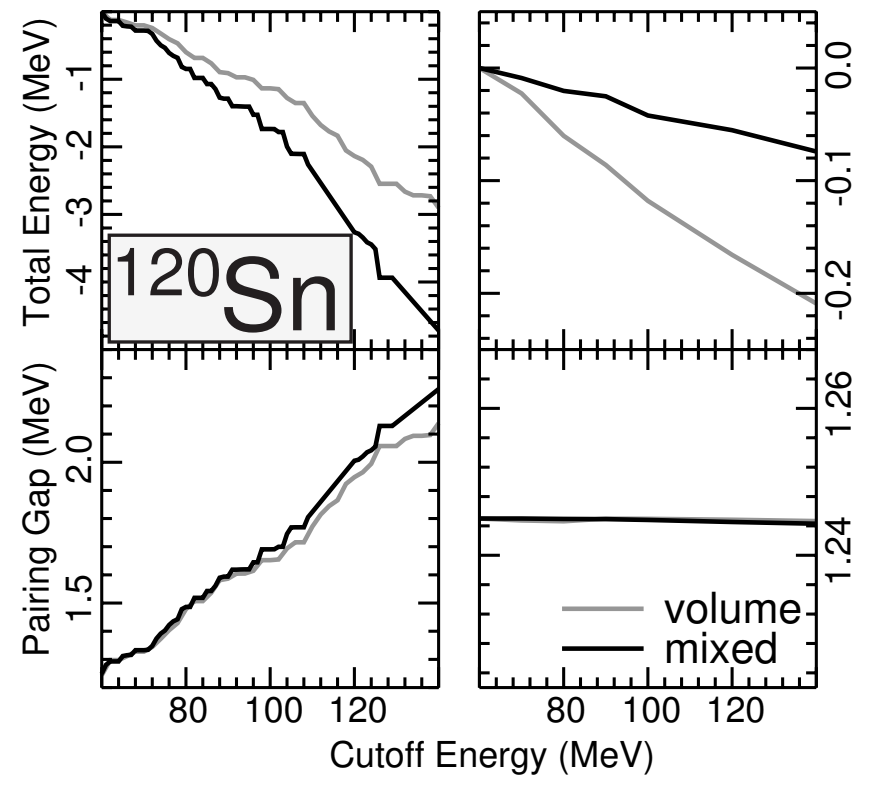

FIG. 2: Total energy (top) and neutron pairing gap (bottom) in ${ }^{120} \mathrm{Sn}$ without (left) and with (right) pairing renormalization applied. Results are shown for volume (gray) and mixed (black) pairing. The total energy is plotted relative to the values obtained for the cutoff energy of $\epsilon_{\text {cut }}=60 \mathrm{MeV}$.

- HFBTHO 26 - diagonalizes the HFB problem in the axially symmetric transformed harmonic oscillator (HO) basis. Unless stated otherwise, we use $N_{o s c}=20 \mathrm{HO}$ shells in the basis.

In our calculations, we use the SLy4 28] and SkP [12] parameterizations of the Skyrme functional in the $\mathrm{p}-\mathrm{h}$ channel and the contact density-dependent force in the p-p channel, which leads to the pairing energy density of the form:

$$
\begin{aligned}
\mathcal{H}_{\text {pair }}(\mathbf{r}) & =\frac{1}{2} g(\mathbf{r}) \tilde{\rho}(\mathbf{r})^{2}= \\
& =\frac{1}{2} V_{0}\left[1-V_{1}\left(\frac{\rho(\mathbf{r})}{\rho_{0}}\right)\right] \tilde{\rho}(\mathbf{r})^{2},
\end{aligned}
$$

where $\rho_{0}=0.16 \mathrm{fm}^{-3}$. For $V_{1}=0$ the resulting pairing interaction is called volume pairing, while $V_{1}=1 / 2$ corresponds to the so-called mixed pairing prescription (Ref. 29] and references quoted therein).

\section{B. Pairing Renormalization}

Figure 2illustrates the importance of the pairing renormalization procedure in the case of ${ }^{120} \mathrm{Sn}$. Due to the constraint (2.2) on the pairing strength, the neutron average pairing gap stays by definition constant, while the resulting total energy changes with the cutoff energy by a few hundred $\mathrm{keV}$. On the other hand, without pairing renormalization applied, the total energy and the average

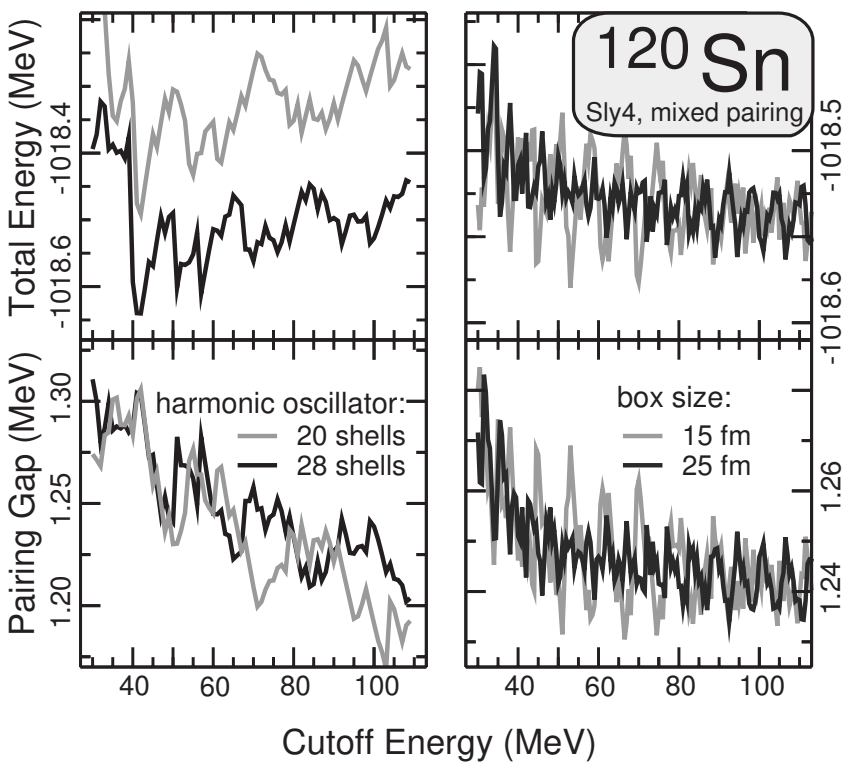

FIG. 3: Total energy (top) and neutron pairing gap (bottom) in ${ }^{120} \mathrm{Sn}$ for the two values of $N_{\text {osc }}$ (left) or using two box sizes (right). Calculations were performed using the mixed pairing interaction.

neutron gap vary significantly with increasing dimension of the quasiparticle space. In this case, the total energy changes by several $\mathrm{MeV}$.

\section{Pairing Regularization}

The total energy and the average neutron pairing gap in ${ }^{120} \mathrm{Sn}$ are shown in Fig. 3 after applying the pairing regularization procedure. The pairing strength $V_{0}$ is kept constant; it reproduces the neutron pairing gap for ${ }^{120} \mathrm{Sn}$ at the cutoff energy of $\epsilon_{\text {cut }}=60 \mathrm{MeV}$.

In the left panels of Fig. 3. we show results obtained in the $\mathrm{HO}$ basis, while the results from the solution of the HFB equations in coordinate space are displayed in the right panels. One can correlate the coordinate-space and HO representations by introducing an 'effective box size' $R \approx \sqrt{2 N_{o s c} \hbar / m \omega}[\underline{8}$. Using this formula, the basis of 20 HO shells corresponds to a box radius of about $14.5 \mathrm{fm}$. Figure 3 demonstrates that the regularization procedure is stable with respect to the cutoff energy. Moreover, one obtains reasonable results already for fairly low cutoff energies of about $40 \mathrm{MeV}$. The variations in the total energy in coordinate-space calculations do not exceed $40 \mathrm{keV}$, while they are about $150 \mathrm{keV}$ in the HO expansion. The latter number does not decrease significantly with $N_{\text {osc }}$.

The differences in applying the pairing regularization procedure in the coordinate-space and $\mathrm{HO}$ calculations can be explained by the different way the quasiparticle space is expanded in both approaches. The particle density $\rho$ is defined by the lower components of the quasi- 


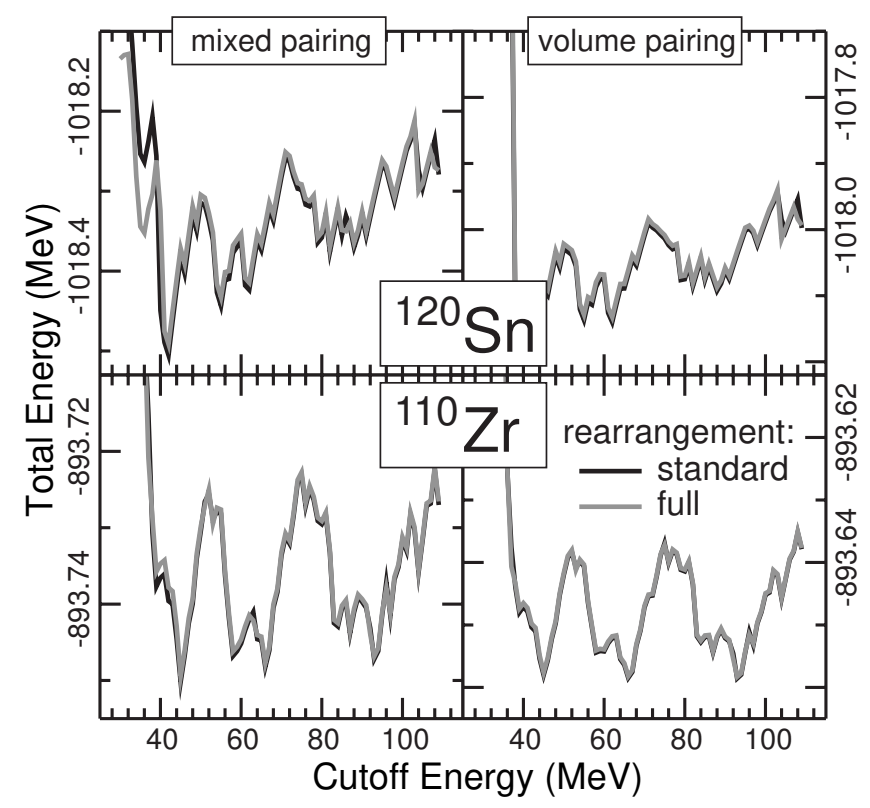

FIG. 4: Total energy of spherical ${ }^{120} \mathrm{Sn}$ (top) and deformed ${ }^{110} \mathrm{Zr}$ (bottom) obtained with pairing regularization (black lines) for mixed pairing (left) and volume pairing (right). The results obtained without the rearrangement terms resulting from the variation of $k_{\text {cut }}$ and $k_{F}$ in Eq. (2.12) are also shown using (gray lines).

particle wave functions, which are localized within the nuclear interior. On the other hand, the abnormal density is defined by the products of the upper and lower components of the quasiparticle wave function. For the quasiparticle energies that are greater than the modulus of the chemical potential, the upper components of the quasiparticle wave function are not localized. Therefore, contrary to the normal density, the abnormal density strongly depends on the completeness of the s.p. basis outside the nuclear interior.

In the coordinate-space calculations, the box boundary conditions provide discretization of the spectrum for the quasiparticle continuum states that are not localized. On the other hand, all the HO basis states are localized. Results of stability with respect to the cutoff energy for the coordinate-space and HO calculations are, therefore, different. As far as the description of nonlocalized states is concerned, the coordinate-space method is superior over the HO expansion method.

Fluctuations in the total energy shown in Fig. 3 coincide with $2 j+1$-folded degenerate angular-momentum multiplets of states in spherical nuclei that enter the pairing window with increasing cutoff energy. This can be confirmed by performing a similar analysis for a deformed nucleus where the magnetic degeneracy is lifted. Such results are shown in Fig. [4 for deformed ${ }^{110} \mathrm{Zr}$ in comparison with spherical ${ }^{120} \mathrm{Sn}$. One can see that the fluctuations of the total energy in ${ }^{110} \mathrm{Zr}$ are down to about $40 \mathrm{keV}$.

The steep increase of the total energy at the cutoff en-

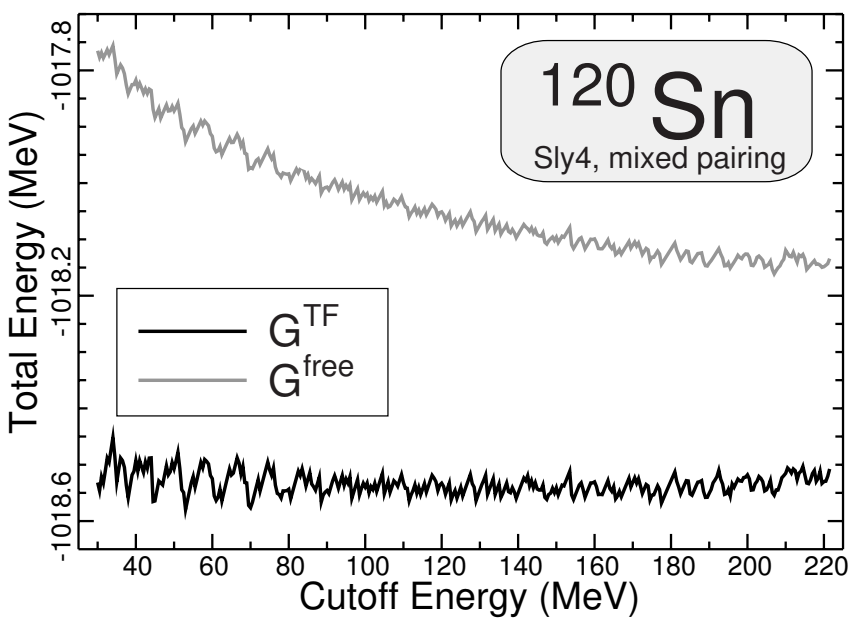

FIG. 5: Two pairing regularization schemes applied to the case of ${ }^{120} \mathrm{Sn}$ : the Thomas-Fermi approximation [19] (black line) and the free particle Green's function [13] (gray line). Coordinate-space calculations were performed in a $15 \mathrm{fm}$ box.

ergies below $30 \mathrm{MeV}$ results from neglecting quasiparticle states with significant occupation probability. This effect is more severe for the mixed-pairing than for volumepairing calculations due to the surface-peaked character of mixed pairing fields. On the other hand, the stability with respect to the cutoff energy is similar in both cases.

We have also tested the importance of the rearrangement terms arising as a result of the regularization procedure. The gray lines in Fig. 4 show results obtained without taking into account the second and third term of Eq. (2.12). These terms lead to changes in the total energy of a few $\mathrm{keV}$ and can be safely neglected.

Finally, we have tested the Thomas-Fermi approximation used in the pairing regularization procedure. Instead of adopting the Thomas-Fermi ansatz, one can perform regularization using the free particle Green's function [13]. As illustrated in Fig. [5] the convergence of the latter method is very slow; the Thomas-Fermi method is clearly superior.

\section{A link between the pairing renormalization and regularization procedures}

The renormalized and regularized pairing calculations are based, in fact, on two different effective interactions. Consequently, their results should be comparable only as much as their effective pairing strengths $g_{\text {eff }}$ are similar. By expanding Eq. (2.11) at very high cutoff energies $\left(k_{F} / k_{\text {cut }}<<1\right)$, one obtains:

$$
g_{\text {eff }}(\mathbf{r}) \approx\left(1-\frac{M^{*}(\mathbf{r}) g(\mathbf{r})}{2 \pi^{2} \hbar^{2}} k_{\mathrm{cut}}(\mathbf{r})\right)^{-1} g(\mathbf{r}),
$$

which has the form of $g_{e f f}=\alpha g$. For the volume pairing, the proportionality factor $\alpha$ is $\rho$-dependent only through 


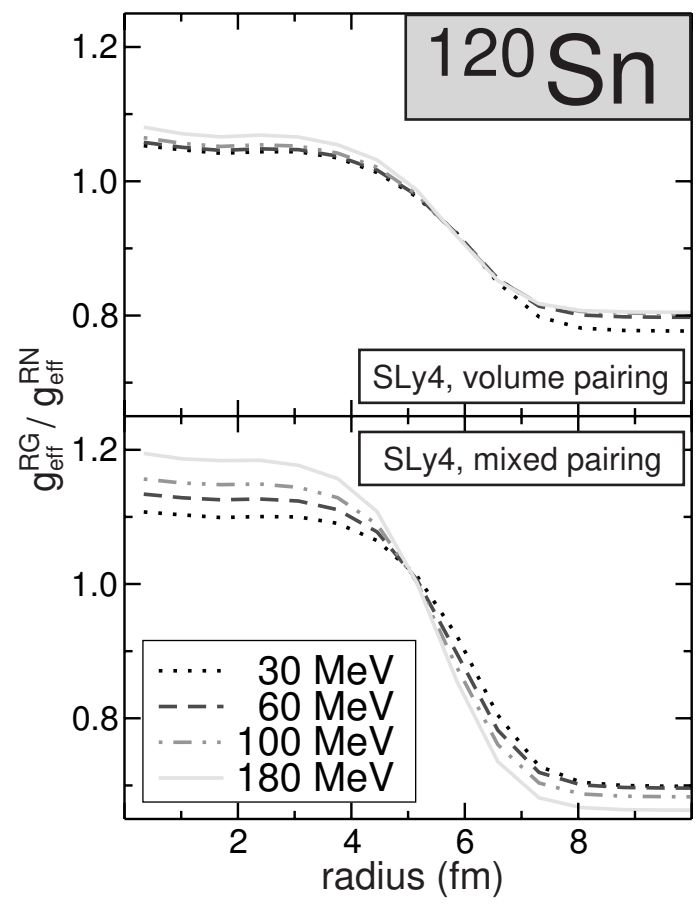

FIG. 6: Ratio between the effective pairing strengths for pairing regularization and renormalization, $g_{\text {eff }}^{R G} / g_{\text {eff }}^{R N}$, for the volume (upper panel) and mixed pairing (lower panel) in ${ }^{120} \mathrm{Sn}$ for several values of $\epsilon_{\text {cut }}$.

the weak density dependence of the effective mass $M^{*}$. On the other hand, for the mixed pairing, it also depends on $\rho$ through the density dependence of $g$. Therefore, while for the volume pairing the renormalization procedure may be considered as a fair approximation to the regularization scheme, this is not the case for the mixed pairing, or - more generally - for any density-dependent pairing. Still, this approximate equality of the effective pairing strengths for the pairing regularization and renormalization is an explanation of the remarkable stability of the total energy in phenomenological pairing renormalization treatment (see Fig. 21), and it also explains why results obtained for the volume pairing are more stable than those in the mixed pairing variant.

This effect can be clearly seen in Fig. 6] The ratio between the effective pairing strengths in the regularization and renormalization methods is much closer to unity for the volume pairing than for the mixed pairing in the region of space, where the pairing energy density is maximal.

\section{COMPARISON BETWEEN PAIRING RENORMALIZATION AND REGULARIZATION PROCEDURES}

In this section, we present a comparison between pairing renormalization and regularization procedures ap- plied to a large number of nuclei. As representative results, we discuss results obtained for the drip-to-drip line isotopic chains of spherical Sn nuclei as well as for deformed Dy nuclei. Calculations are performed for the volume and/or mixed pairing interactions by using the $\mathrm{HFB}+\mathrm{THO}$ approach.

\section{A. Spherical Nuclei}

Figure[7 displays differences between the pairing renormalization and regularization procedures for the Sn isotopes. Calculations are performed with both volume and mixed pairing interactions. For the two-neutron separation energies, the maximum difference between the renormalization and regularization schemes is about 100 (300) $\mathrm{keV}$ for the volume (mixed) pairing. In the neutron gap, the corresponding difference is about $50(100) \mathrm{keV}$, and in nuclear radii (not displayed) it is practically negligible (about $0.01 \mathrm{fm}$ ). The largest differences show up in pairing energies - about 1 (3) $\mathrm{MeV}$ for the volume (mixed) pairing; however, total energy differences are much smaller - about 400 (800) keV.

Analyzing the total energies obtained in both methods, Fig. 7(a), one can see that the pairing renormalization procedure gives systematically more binding. The differences are negligible for stable nuclei and nuclei near the proton drip line. They increase in mid-shell nuclei near the two-neutron drip line where the pairing effects are the largest, and then decrease towards the closedshell nucleus ${ }^{176} \mathrm{Sn}$ located just at the two-neutron drip line. In general, both procedures give more similar results in the case of volume pairing than in the case of mixed pairing.

Recently, the pairing regularization procedure has been analyzed in the context of relativistic mean-field approximation [23]. In order to simulate the finite range contribution to the nuclear matter pairing gap coming from the Gogny pairing force, it was necessary to introduce strong density dependence in the pairing strength of the contact interaction.

Using the regularization procedure and calculating the Sn chain with both volume and newly constructed (surface) contact interaction, the authors of Ref. 23] have found differences in pairing energies of the order of $20 \mathrm{MeV}$ in the neutron-rich nuclei around ${ }^{148} \mathrm{Sn}$. In our work, for the same nuclei, the differences in pairing energies between volume and mixed pairing variants do not exceed $2.6 \mathrm{MeV}$. This comparison shows that the densitydependent contact interaction proposed in Ref. 23] is questionable for finite nuclei, despite its agreement with the finite-range Gogny pairing force in the infinite nuclear matter. 

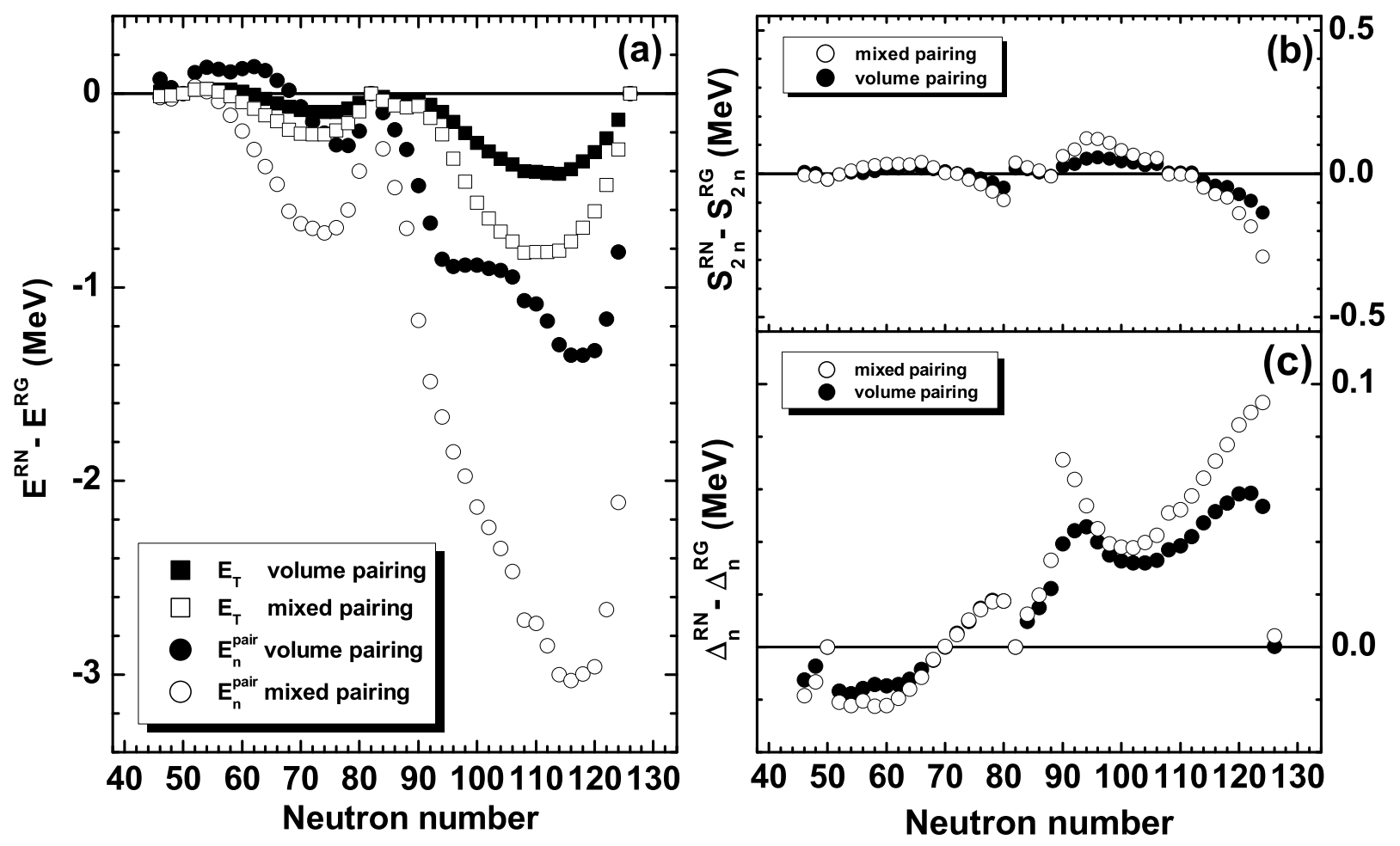

FIG. 7: Differences between pairing renormalization (RN) and regularization (RG) procedures for (a) total energies and neutron pairing energies, (b) two neutron separation energies, and (c) the average neutron gaps. The HFB+THO calculations are performed for the chain of the spherical Sn isotopes using the SkP Skyrme parameterization.

\section{B. Deformed Nuclei}

We applied the pairing renormalization and regularization procedures for the chain of deformed Dy isotopes. Differences between both sets of results are shown in Fig. 8 We show only the results with the mixed pairing, since, as in the spherical nuclei, the differences between both procedures are larger in this case.

As seen in Fig. 8] (c), most of the nuclei considered are well deformed, and the deformations are practically the same within both procedures. Despite the fact that the maximum difference in the pairing energy is around $3 \mathrm{MeV}$ (not shown), other quantities are very similar. The maximum difference in the total energy is about $360 \mathrm{keV}$, in the two-neutron separation energy $-160 \mathrm{keV}$, in the pairing gaps $-110 \mathrm{keV}$, and in the rms radii (not shown) - less than $0.005 \mathrm{fm}$.

\section{SUMMARY AND CONCLUSIONS}

In this work, we investigated the pairing regularization method using the s.p. Green's function in the ThomasFermi approximation and found it to be very suitable for a description of spherical and deformed nuclei. We checked the stability of the method with respect to the cutoff energy and found fluctuations in the total energy below $200 \mathrm{keV}$. Fluctuations coming from the method it- self do not exceed $50 \mathrm{keV}$ for the cutoff energy as low as $30 \mathrm{MeV}$. However, if a still lower cutoff energy is assumed, the Thomas-Fermi approximation to the s.p. Green's function may no longer be valid.

We found that the differences between pairing renormalization and regularization procedures for volume and mixed pairing are rather small. Therefore, we conclude that physical conclusions previously obtained within the pairing renormalization scheme remain valid. Nevertheless, we believe that the theoretical motivation and simplicity of the regularization method is preferred to a phenomenological renormalization scheme.

\section{Acknowledgments}

Discussions with Aurel Bulgac are gratefully acknowledged. This work was supported in part by the U.S. Department of Energy under Contract Nos. DEFG02-96ER40963 (University of Tennessee), DE-AC0500OR22725 with UT-Battelle, LLC (Oak Ridge National Laboratory), by the National Nuclear Security Administration under the Stewardship Science Academic Alliances program through DOE Research Grant DE-FG0303NA00083; by the Polish Committee for Scientific Research (KBN) under contract N0. 1 P03B 05927 and by the Foundation for Polish Science (FNP). 

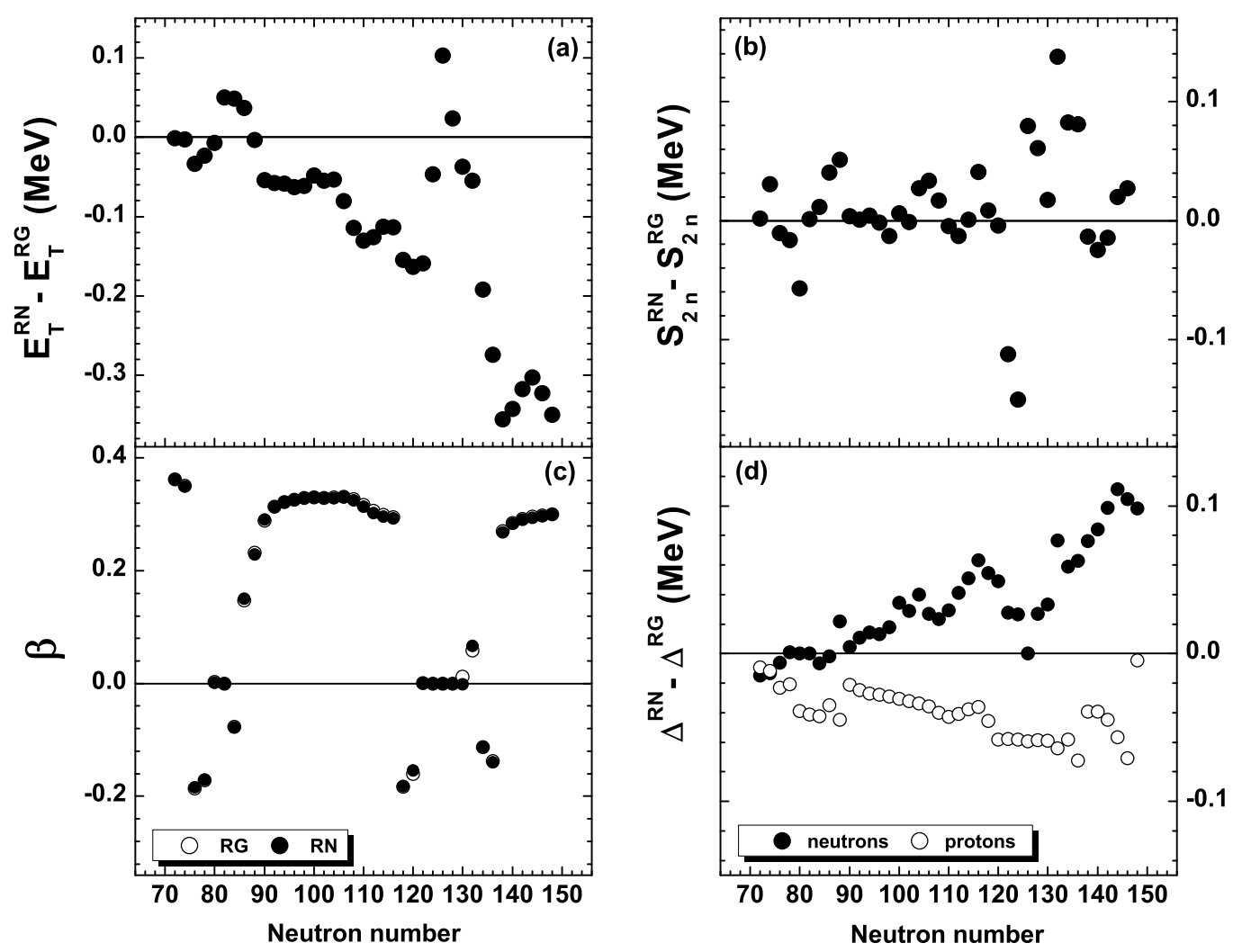

FIG. 8: Similar to Fig. [7 except for the deformed Dy isotopes. Quadrupole deformations are displayed in panel (c). Mixed pairing interaction was used.

\section{APPENDIX A: DIVERGENCE IN THE ABNORMAL DENSITY}

In the DFT-HFB approach, the starting point is the Energy Density Functional (EDF) $\mathcal{H}[\rho, \tilde{\rho}]$, where $\rho$ is the particle density and $\tilde{\rho}$ is the abnormal density:

$$
\begin{aligned}
& \rho\left(\mathbf{r}_{\mathbf{2}} \sigma_{2} \tau_{2}, \mathbf{r}_{1} \sigma_{1} \tau_{1}\right)=\left\langle\Phi\left|a_{\mathbf{r}_{1} \sigma_{1} \tau_{1}}^{\dagger} a_{\mathbf{r}_{2} \sigma_{2} \tau_{2}}\right| \Phi\right\rangle \\
& \tilde{\rho}\left(\mathbf{r}_{\mathbf{2}} \sigma_{2} \tau_{2}, \mathbf{r}_{1} \sigma_{1} \tau_{1}\right)=-2 \sigma_{1}\left\langle\Phi\left|a_{\mathbf{r}_{1}-\sigma_{1} \tau_{1}} a_{\mathbf{r}_{2} \sigma_{2} \tau_{2}}\right| \Phi\right\rangle,
\end{aligned}
$$

where $a$ and $a^{\dagger}$ are the particle annihilation and creation operators, respectively, and $|\Phi\rangle$ is the HFB state. In the following, we assume that $|\Phi\rangle$ is a product of the neutron and proton states, $\left|\Phi_{\nu}\right\rangle\left|\Phi_{\pi}\right\rangle$. Therefore, the neutron and proton wave functions are not coupled, and in the notation below we can, for simplicity, omit the isospin index with the understanding that all equations are separately valid for neutrons and protons.

For the HFB state $|\Phi\rangle$, the particle and abnormal densities can be written as 12:

$$
\begin{aligned}
& \rho\left(\mathbf{r}_{\mathbf{2}} \sigma_{2}, \mathbf{r}_{\mathbf{1}} \sigma_{1}\right)=\sum_{E_{i}>0} \varphi_{2 i}\left(\mathbf{r}_{\mathbf{2}} \sigma_{2}\right) \varphi_{2 i}^{*}\left(\mathbf{r}_{\mathbf{1}} \sigma_{1}\right), \\
& \tilde{\rho}\left(\mathbf{r}_{\mathbf{2}} \sigma_{2}, \mathbf{r}_{\mathbf{1}} \sigma_{1}\right)=-\sum_{E_{i}>0} \varphi_{2 i}\left(\mathbf{r}_{\mathbf{2}} \sigma_{2}\right) \varphi_{1 i}^{*}\left(\mathbf{r}_{\mathbf{1}} \sigma_{1}\right),
\end{aligned}
$$

where the two-component quasiparticle wave function $\varphi$ is the solution of the HFB equation:

$$
\begin{aligned}
& \sum_{\sigma_{1}} \int d^{3} \mathbf{r}_{\mathbf{1}}\left[\begin{array}{cc}
h_{\mu}\left(\mathbf{r}_{\mathbf{2}} \sigma_{2}, \mathbf{r}_{\mathbf{1}} \sigma_{1}\right) & \tilde{h}\left(\mathbf{r}_{\mathbf{2}} \sigma_{2}, \mathbf{r}_{\mathbf{1}} \sigma_{1}\right) \\
\tilde{h}\left(\mathbf{r}_{\mathbf{2}} \sigma_{2}, \mathbf{r}_{\mathbf{1}} \sigma_{1}\right) & -h_{\mu}\left(\mathbf{r}_{\mathbf{2}} \sigma_{2}, \mathbf{r}_{\mathbf{1}} \sigma_{1}\right)
\end{array}\right] \times \\
& \times\left[\begin{array}{l}
\varphi_{1 i}\left(\mathbf{r}_{\mathbf{1}} \sigma_{1}\right) \\
\varphi_{2 i}\left(\mathbf{r}_{\mathbf{1}} \sigma_{1}\right)
\end{array}\right]=E_{i}\left[\begin{array}{c}
\varphi_{1 i}\left(\mathbf{r}_{\mathbf{2}} \sigma_{2}\right) \\
\varphi_{2 i}\left(\mathbf{r}_{\mathbf{2}} \sigma_{2}\right)
\end{array}\right],
\end{aligned}
$$

for a given quasiparticle energy $E_{i}$.

The HFB equations are a result of variational minimization of the energy density functional $\mathcal{H}[\rho, \tilde{\rho}]$ with the constraint of the mean value of particles kept constant:

$$
\left.\delta \mathcal{H}\right|_{\langle\hat{N}\rangle=N}=0 .
$$

This condition defines the s.p. Hamiltonian $h_{\mu}$ and the pairing Hamiltonian $\tilde{h}$ in the HFB equations A5:

$$
\begin{aligned}
& h_{\mu}\left(\mathbf{r}_{\mathbf{2}} \sigma_{2}, \mathbf{r}_{1} \sigma_{1}\right)=\frac{\delta \mathcal{H}[\rho, \tilde{\rho}]}{\delta \rho\left(\mathbf{r}_{1} \sigma_{1}, \mathbf{r}_{\mathbf{2}} \sigma_{2}\right)}-\mu \\
& =-\nabla_{\mathbf{r}_{2}} \frac{\hbar^{2}}{2 M^{*}\left(\mathbf{r}_{\mathbf{2}} \sigma_{2}, \mathbf{r}_{\mathbf{1}} \sigma_{1}\right)} \nabla_{\mathbf{r}_{\mathbf{1}}}+U\left(\mathbf{r}_{\mathbf{2}} \sigma_{2}, \mathbf{r}_{\mathbf{1}} \sigma_{1}\right)-\mu \\
& \quad \tilde{h}\left(\mathbf{r}_{\mathbf{2}} \sigma_{2}, \mathbf{r}_{\mathbf{1}} \sigma_{1}\right)=\frac{\delta \mathcal{H}[\rho, \tilde{\rho}]}{\delta \tilde{\rho}\left(\mathbf{r}_{\mathbf{1}} \sigma_{2}, \mathbf{r}_{\mathbf{2}} \sigma_{2}\right)}
\end{aligned}
$$

where $M^{*}$ is the effective mass and $U$ is the self-consistent mean-field potential. In the following derivations, the 
spin-orbit term is omitted as unimportant in the regularization scheme, although it is, of course, always included in calculations.
By multiplying the HFB equations (A5) by vector $\left[\varphi_{2 i}^{*},-\varphi_{1 i}^{*}\right]$, integrating over coordinates and summing over all the positive energy HFB solutions, one obtains:

$$
\begin{aligned}
& \sum_{E_{i}>0, \sigma_{2}} E_{i} \int d^{3} \mathbf{r}_{\mathbf{2}}\left[\varphi_{2 i}^{*}\left(\mathbf{r}_{\mathbf{2}} \sigma_{2}\right),-\varphi_{1 i}^{*}\left(\mathbf{r}_{\mathbf{2}} \sigma_{2}\right)\right]\left[\begin{array}{l}
\varphi_{1 i}\left(\mathbf{r}_{\mathbf{2}} \sigma_{2}\right) \\
\varphi_{2 i}\left(\mathbf{r}_{\mathbf{2}} \sigma_{2}\right)
\end{array}\right]= \\
& \quad=\sum_{E_{i}>0, \sigma_{1} \sigma_{2}} \iint d^{3} \mathbf{r}_{\mathbf{1}} d^{3} \mathbf{r}_{\mathbf{2}}\left[\varphi_{2 i}^{*}\left(\mathbf{r}_{\mathbf{2}} \sigma_{2}\right),-\varphi_{1 i}^{*}\left(\mathbf{r}_{\mathbf{2}} \sigma_{2}\right)\right]\left[\begin{array}{cc}
h_{\mu}\left(\mathbf{r}_{\mathbf{2}} \sigma_{2}, \mathbf{r}_{1} \sigma_{1}\right) & \tilde{h}\left(\mathbf{r}_{\mathbf{2}} \sigma_{2}, \mathbf{r}_{1} \sigma_{1}\right) \\
\tilde{h}\left(\mathbf{r}_{\mathbf{2}} \sigma_{2}, \mathbf{r}_{\mathbf{1}} \sigma_{1}\right) & -h_{\mu}\left(\mathbf{r}_{\mathbf{2}} \sigma_{2}, \mathbf{r}_{\mathbf{1}} \sigma_{1}\right)
\end{array}\right]\left[\begin{array}{l}
\varphi_{1 i}\left(\mathbf{r}_{\mathbf{1}} \sigma_{1}\right) \\
\varphi_{2 i}\left(\mathbf{r}_{\mathbf{1}} \sigma_{1}\right)
\end{array}\right]
\end{aligned}
$$

i.e.,

$$
\begin{aligned}
& \sum_{E_{i}>0, \sigma_{1}} E_{i} \int d^{3} \mathbf{r}_{\mathbf{1}}\left\{\varphi_{2 i}^{*}\left(\mathbf{r}_{\mathbf{1}} \sigma_{1}\right) \varphi_{1 i}\left(\mathbf{r}_{\mathbf{1}} \sigma_{1}\right)-\varphi_{1 i}^{*}\left(\mathbf{r}_{\mathbf{1}} \sigma_{1}\right) \varphi_{2 i}\left(\mathbf{r}_{\mathbf{1}} \sigma_{1}\right)\right\}= \\
& =\sum_{E_{i}>0, \sigma_{1} \sigma_{2}} \iint d^{3} \mathbf{r}_{\mathbf{1}} d^{3} \mathbf{r}_{\mathbf{2}}\left\{\varphi_{2 i}^{*}\left(\mathbf{r}_{\mathbf{2}} \sigma_{2}\right) h_{\mu}\left(\mathbf{r}_{\mathbf{2}} \sigma_{2}, \mathbf{r}_{\mathbf{1}} \sigma_{1}\right) \varphi_{1 i}\left(\mathbf{r}_{\mathbf{1}} \sigma_{1}\right)+\varphi_{2 i}^{*}\left(\mathbf{r}_{\mathbf{2}} \sigma_{2}\right) \tilde{h}\left(\mathbf{r}_{\mathbf{2}} \sigma_{2}, \mathbf{r}_{\mathbf{1}} \sigma_{1}\right) \varphi_{2 i}\left(\mathbf{r}_{\mathbf{1}} \sigma_{1}\right)+\right. \\
& \left.\quad+\varphi_{1 i}^{*}\left(\mathbf{r}_{\mathbf{2}} \sigma_{2}\right) h_{\mu}\left(\mathbf{r}_{\mathbf{2}} \sigma_{2}, \mathbf{r}_{\mathbf{1}} \sigma_{1}\right) \varphi_{2 i}\left(\mathbf{r}_{\mathbf{1}} \sigma_{1}\right)-\varphi_{1 i}^{*}\left(\mathbf{r}_{\mathbf{2}} \sigma_{2}\right) \tilde{h}\left(\mathbf{r}_{\mathbf{2}} \sigma_{2}, \mathbf{r}_{\mathbf{1}} \sigma_{1}\right) \varphi_{1 i}\left(\mathbf{r}_{\mathbf{1}} \sigma_{1}\right)\right\} .
\end{aligned}
$$

Since for every HFB solution $\left(\left[\varphi_{1 i}, \varphi_{2 i}\right], E_{i}\right)$ there exists also an orthogonal solution $\left(\left[\varphi_{2 i},-\varphi_{1 i}\right],-E_{i}\right)$, the left-hand side of Eq. (A10) vanishes as a sum over scalar products of orthogonal wave functions.

For local and spin-independent Hamiltonians $h_{\mu}$ and $\tilde{h}$, Eqs. (A8) and (A8) read

$$
\begin{aligned}
h_{\mu}\left(\mathbf{r}_{\mathbf{2}} \sigma_{2}, \mathbf{r}_{\mathbf{1}} \sigma_{1}\right) & =-\nabla_{\mathbf{r}_{\mathbf{2}}} \frac{\hbar^{2}}{2 M^{*}\left(\mathbf{r}_{\mathbf{2}}\right)} \delta\left(\mathbf{r}_{\mathbf{2}}-\mathbf{r}_{\mathbf{1}}\right) \delta_{\sigma_{2}, \sigma_{1}} \nabla_{\mathbf{r}_{\mathbf{1}}}+\left(U\left(\mathbf{r}_{\mathbf{2}}\right)-\mu\right) \delta\left(\mathbf{r}_{\mathbf{2}}-\mathbf{r}_{\mathbf{1}}\right) \delta_{\sigma_{2}, \sigma_{1}}, \\
\tilde{h}\left(\mathbf{r}_{\mathbf{2}} \sigma_{2}, \mathbf{r}_{\mathbf{1}} \sigma_{1}\right) & =\tilde{h}\left(\mathbf{r}_{\mathbf{2}}\right) \delta\left(\mathbf{r}_{\mathbf{2}}-\mathbf{r}_{\mathbf{1}}\right) \delta_{\sigma_{2}, \sigma_{1}} .
\end{aligned}
$$

Note that for an attractive pairing force, the local pairing potential $\tilde{h}(\mathbf{r})=-\Delta(\mathbf{r})$ is negative, where $\Delta(\mathbf{r})$ is the standard position-dependent pairing gap. By defining function $\mathcal{F}_{\epsilon_{\mathrm{cut}}}$ as

$$
\sum_{E_{i}>0, \sigma}\left[\varphi_{1 i}\left(\mathbf{r}_{\mathbf{2}} \sigma\right) \varphi_{1 i}^{*}\left(\mathbf{r}_{\mathbf{1}} \sigma\right)+\varphi_{2 i}\left(\mathbf{r}_{\mathbf{2}} \sigma\right) \varphi_{2 i}^{*}\left(\mathbf{r}_{\mathbf{1}} \sigma\right)\right]=\mathcal{F}_{\epsilon_{\mathrm{cut}}}\left(\mathbf{r}_{\mathbf{2}}-\mathbf{r}_{\mathbf{1}}\right) .
$$

and using expression (A4) for the abnormal density, one obtains after integrating the kinetic-energy term by parts:

$$
\begin{aligned}
0 & =-\int d^{3} \mathbf{r}_{\mathbf{1}} d^{3} \mathbf{r}_{\mathbf{2}} \delta\left(\mathbf{r}_{\mathbf{2}}-\mathbf{r}_{\mathbf{1}}\right)\left[\tilde{h}\left(\mathbf{r}_{\mathbf{2}}\right)\left[\mathcal{F}_{\epsilon_{\mathrm{cut}}}\left(\mathbf{r}_{\mathbf{2}}-\mathbf{r}_{\mathbf{1}}\right)-2 \rho\left(\mathbf{r}_{\mathbf{2}}, \mathbf{r}_{1}\right)\right]+\left(\frac{\hbar^{2}}{2 M^{*}} \nabla_{\mathbf{r}_{\mathbf{2}}} \nabla_{\mathbf{r}_{1}}+U\left(\mathbf{r}_{\mathbf{2}}\right)-\mu\right) 2 \tilde{\rho}\left(\mathbf{r}_{1}, \mathbf{r}_{2}\right)\right]= \\
& =-\int d^{3} \mathbf{r} d^{3} \mathbf{x} \delta(\mathbf{x})\left[\tilde{h}(\mathbf{r})\left[\mathcal{F}_{\epsilon_{\mathrm{cut}}}(\mathbf{x})-2 \rho(\mathbf{r}, \mathbf{r})\right]+\left(\frac{\hbar^{2}}{2 M^{*}}\left(\frac{1}{4} \nabla_{\mathbf{r}}^{2}-\nabla_{\mathbf{x}}^{2}\right)+U(\mathbf{r})-\mu\right) 2 \tilde{\rho}(\mathbf{r}-\mathbf{x} / 2, \mathbf{r}+\mathbf{x} / 2)\right],(\mathrm{A} 14)
\end{aligned}
$$

where

$$
\begin{aligned}
& \mathbf{r}=\frac{\mathbf{r}_{1}+\mathbf{r}_{2}}{2}, \\
& \mathbf{x}=\mathbf{r}_{2}-\mathbf{r}_{1},
\end{aligned}
$$

and

$$
\begin{aligned}
& \rho\left(\mathbf{r}_{\mathbf{2}}, \mathbf{r}_{\mathbf{1}}\right)=\sum_{\sigma} \rho\left(\mathbf{r}_{\mathbf{2}} \sigma, \mathbf{r}_{\mathbf{1}} \sigma\right), \\
& \tilde{\rho}\left(\mathbf{r}_{\mathbf{2}}, \mathbf{r}_{\mathbf{1}}\right)=\sum_{\sigma} \tilde{\rho}\left(\mathbf{r}_{\mathbf{2}} \sigma, \mathbf{r}_{\mathbf{1}} \sigma\right) .
\end{aligned}
$$

When the summation over positive quasiparticle energies is extended to infinity, the completeness relation implies that

$$
\mathcal{F}_{\epsilon_{\mathrm{cut}}}\left(\mathbf{r}_{2}-\mathbf{r}_{1}\right)=\delta\left(\mathbf{r}_{2}-\mathbf{r}_{1}\right),
$$

and the only term in Eq. A14 capable of canceling out this singularity is $\nabla_{\mathbf{x}}^{2} \tilde{\rho}(\mathbf{r}-\mathbf{x} / 2, \mathbf{r}+\mathbf{x} / 2)$. Therefore, the Laplacian of the abnormal density $\nabla_{\mathbf{x}}^{2} \tilde{\rho}(\mathbf{r}-\mathbf{x} / 2, \mathbf{r}+\mathbf{x} / 2)$ must be singular at $\mathbf{x}=0$. Moreover, using the expres- 
sion

$$
\nabla^{2} \frac{1}{|\mathbf{r}|}=-4 \pi \delta(\mathbf{r})
$$

it is clear that due to the zero-range pairing interaction abnormal density $\tilde{\rho}$ has an ultraviolet $1 / x$ divergence:

$$
\tilde{\rho}(\mathbf{r}-\mathbf{x} / 2, \mathbf{r}+\mathbf{x} / 2) \sim-\left.\frac{\tilde{h}(\mathbf{r}) M^{*}(\mathbf{r})}{4 \pi \hbar^{2}|\mathbf{x}|}\right|_{\mathbf{x} \rightarrow 0} .
$$

[1] P. Hohenberg and W. Kohn, Phys. Rev. 136, B864 (1964)

[2] W. Kohn and L.J. Sham, Phys. Rev. 140, A1133 (1965)

[3] T.H.R. Skyrme, Phil. Mag. 1, 1043 (1956)

[4] D. Vauterin and D.M Brink, Phys. Rev. C 5, 626 (1972)

[5] E. Perlińska, S.G. Rohozinski, J. Dobaczewski, and W. Nazarewicz, Phys. Rev. C 69, 014316 (2004)

[6] M. Samyn, S. Goriely, P.-H. Heenen, J.M. Pearson, and F. Tondeur, Nucl. Phys. A 700, 142 (2002)

[7] S. Goriely, M. Samyn, P.-H. Heenen, J.M. Pearson, and F. Tondeur, Phys. Rev. C 66, 024326-1 (2002)

[8] J. Dobaczewski, W. Nazarewicz, T.R. Werner, J.F. Berger, C.R. Chinn, and J. Dechargé, Phys. Rev. C 53, 2809 (1996)

[9] J. Dobaczewski, W. Nazarewicz, and P.-G. Reinhard, Nucl. Phys. A 693, 361 (2001)

[10] J. Dechargé and D. Gogny, Phys. Rev. C 21, 1568 (1980)

[11] R.R. Chasman, Phys. Rev. C 14, 1935 (1976)

[12] J. Dobaczewski, H. Flocard, and J. Treiner, Nucl. Phys. A 422, 103 (1984)

[13] H. Esbensen, G.F. Bertsch, and K. Hencken, Phys. Rev. C 56, 3054 (1997)

[14] M. Marini, F. Pistolesi, and G.C. Strinati, Eur. Phys. J. B 1, 151 (1998)

[15] T. Papenbrock and G.F. Bertsch, Phys. Rev. C 59, 2052
(1999)

[16] G. Bruun, Y. Castin, R. Dum, and K. Burnett, Eur. Phys. J. D 7, 433 (1999)

[17] A. Bulgac, nucl-th/9907088

[18] A. Bulgac and Y. Yu, nucl-th/0109083

[19] A. Bulgac and Y. Yu, Phys. Rev. Lett. 88, 042504 (2002)

[20] A. Bulgac, Phys. Rev. C 65, 051305(R) (2002)

[21] Y. Yu and A. Bulgac, Phys. Rev. Lett. 90, 222501 (2003)

[22] A. Bulgac and Y. Yu, Int. J. Mod. Phys. E 13, 147 (2004)

[23] T. Nikšić, P. Ring, and D. Vretenar, Phys. Rev. C 71, $044320(2005)$

[24] M. Grasso and M. Urban, Phys. Rev. A 68, 033610 (2003)

[25] P. Ring and P. Schuck, The Nuclear Many-Body Problem. Springer-Verlag, New York (1980)

[26] M.V. Stoitsov, J. Dobaczewski, W. Nazarewicz, and P. Ring, Comput. Phys. Commun. 167, 43 (2005)

[27] K. Benneceur and J. Dobaczewski, Comput. Phys. Commun. 168, 96 (2005)

[28] E. Chabanat, P. Bonche, P. Haensel, J. Meyer, and F. Schaeffer, Nucl. Phys. A 635, 231 (1998)

[29] J. Dobaczewski, W. Nazarewicz, and M.V. Stoitsov, Eur. Phys. J. A 15, 21 (2002) 